\title{
Early Detection of Airborne Inoculum of Magnaporthe oryzae in Turfgrass Fields Using a Quantitative LAMP Assay
}

Caterina Villari, Department of Plant Pathology, The Ohio State University, Columbus 43210; Walter F. Mahaffee, Horticultural Crops Research Laboratory, United States Department of Agriculture-Agricultural Research Service (USDA-ARS), Corvallis, OR 97330; Thomas K. Mitchell, Department of Plant Pathology, The Ohio State University; Kerry F. Pedley, and Michael L. Pieck, Foreign Disease-Weed Science Research Unit, USDA-ARS, Fort Detrick, MD 21702; and Francesca Peduto Hand, Department of Plant Pathology, The Ohio State University, Columbus, OH 43210

\begin{abstract}
Gray leaf spot (GLS) is a destructive disease of perennial ryegrass caused by a host specific pathotype of the ascomycete Magnaporthe oryzae. Early diagnosis is crucial for effective disease management and the implementation of Integrated Pest Management practices. However, a rapid protocol for the detection of low levels of airborne inoculum is still missing. We developed a pathogen-specific quantitative loop-mediated isothermal amplification (qLAMP) assay coupled with a spore trap system for rapid detection and quantification of airborne inoculum of the $M$. oryzae perennial ryegrass pathotype, and tested its suitability for implementation in GLS-infected turfgrass fields. In summer 2015, two

perennial ryegrass plots were artificially inoculated with the pathogen, with four continuously running custom impaction spore traps placed in each plot. Sampling units were replaced daily and tested with the developed qLAMP assay, while plots were monitored for symptom development. Results confirmed that the qLAMP assay-trap system was able to detect as few as 10 conidia up to 12 days before symptoms developed in the field. LAMP technology is particularly appropriate for field implementation by nontechnical users, and has the potential to be a powerful decision support tool to guide timing of fungicide applications for GLS management.
\end{abstract}

Despite turfgrass managers recognizing that increased environmental stewardship is necessary for their industry to thrive, the implementation of Integrated Pest Management (IPM) in turfgrass systems still poses a unique challenge in spite of this crop being highly monitored and managed (Held and Potter 2012). A valuable help to guide disease management decisions, and hence IPM implementation, is the early detection of pathogen inoculum. In the case of airborne inoculum, early detection has been used as a driver to initiate and time fungicide application in numerous pathosystems, including potato early blight and grape powdery mildew (Mahaffee 2014). However, turfgrass disease management often deals with sudden and sporadic outbreaks and, for certain diseases, affected turf can be destroyed in only a few days (Uddin et al. 2003c). For early detection to succeed, identification must be completed in a very short period of time; hence, inoculum trapping methods need to be associated with technologies that allow for a rapid and reliable identification of the pathogen.

Gray leaf spot (GLS) is a destructive disease of perennial ryegrass (Lolium perenne L.) caused by the ascomycete Magnaporthe oryzae B. C. Couch (anamorph = Pyricularia oryzae Cavara) (Uddin et al. 2003c), formerly known as M. grisea (T. T. Herbert) M. E. Barr (Couch and Kohn 2002). This pathogen is also very well known as the causal agent of rice (Oryza sativa L.) and wheat (Triticum aestivum L.) blast. Although M. oryzae can infect over 50 plant species (Couch and Kohn 2002), isolates exhibit host specificity (Farman

Corresponding author: F. Peduto Hand; E-mail: hand.81@osu.edu

Disclaimer: The use of trade, firm, or corporation names in this publication is for the information and convenience of the reader. Such use does not constitute an official endorsement or approval by the United States Department of Agriculture or the Agricultural Research Service of any product or service to the exclusion of others that may be suitable.

*The $\boldsymbol{e}$-Xtra logo stands for "electronic extra" and indicates that one supplementary table is published online.

Accepted for publication 7 September 2016.

C 2017 The American Phytopathological Society
2002; Viji et al. 2001) and, despite being morphologically identical (Rossman et al. 1990), they can be grouped in genetically distinct pathotypes (Farman 2002).

Outbreaks of GLS, which causes blighting and quick death of large areas of turf, have recently become a serious but sporadic problem for turfgrass managers in the Midwestern and Northeastern United States (Uddin et al. 2003c). Due to the rapid progression of the disease and high susceptibility of the host, fungicides are currently the best available management tools (Uddin et al. 2003c). Symptoms of GLS are not easily recognizable and can be confused with those of other summer diseases or stress-related disorders (Harmon et al. 2003). Nevertheless, a timely diagnosis is crucial for efficient disease management. In addition to conventional methods that rely on microscopic observation of spores, an immunological assay (Uddin et al. 2003b) and a polymerase chain reaction (PCR)-based assay (Harmon et al. 2003) have been developed for GLS diagnosis. However, these methods require the analysis of symptomatic leaf tissues and might not be sensitive enough when low levels of infection are present or when samples that are still asymptomatic are processed. Given that airborne conidia of $M$. oryzae constitute the primary inoculum for leaf infections early in the growing season, and because GLS develops at visually undetectable levels before triggering a series of secondary infections (Uddin et al. 2003c), timely detection of primary inoculum would be a more useful identification tool. Moreover, the above-mentioned diagnostic assays (Harmon et al. 2003; Uddin et al. 2003b) are not implementable in the field and require analyses to be performed in laboratories.

Loop-mediated isothermal amplification (LAMP) (Notomi et al. 2000) is a highly sensitive molecular technology that has been successfully applied to the detection of several plant pathogens (Elkins et al. 2015; Thiessen et al. 2016; Tomlinson et al. 2013; Villari et al. 2013). Unlike other PCR-based assays, LAMP reactions are isothermal; hence, there is no need for expensive thermal cycling equipment. Besides requiring a reaction time not greater than $1 \mathrm{~h}$ (Notomi et al. 2000), LAMP is also less sensitive to PCR inhibitors, allowing for the use of crude DNA extracts (Kogovšek et al. 2015; Poon et al. 2006; Tomlinson et al. 2007). These traits, in addition to the lack of need for extensive training of the personnel performing the assay (Thiessen et al. 2016; Tomlinson et al. 2013), meet all the major technical and economic requirements for successful implementation in horticultural settings (Lievens and Thomma 2005), and make this technology 
particularly appropriate for field implementation by nontechnical users. At the very least, successful amplifications can be visually observed by a change in turbidity in the reaction tubes (Mori et al. 2001). More recently, however, LAMP has been optimized for visualization using fluorescent assimilating probes that allow for quantitative assays (Kubota et al. 2011) without losing the capability of being run directly on portable devices (Jenkins et al. 2011).

The aims of this study were to (i) develop a quantitative assimilating probe-based LAMP (henceforth, qLAMP) assay for the rapid detection and quantification of airborne inoculum of $M$. oryzae perennial ryegrass pathotype and (ii) test its suitability for implementation in turfgrass fields when coupled with a custom-made impaction spore trap system. This study represents the first step toward the ultimate goal of using a qLAMP assay-trap system as a decision support tool for turf managers and superintendents to guide disease management of GLS and, potentially, other important diseases of turf.

\section{Materials and Methods}

Sampling rod preparation. Stainless steel sampling rods ( 1.1 by $40 \mathrm{~mm}$ ) were coated with vacuum grease (Dow Corning, Midland, MI) and embedded in pairs in the lid of a sterile 15-ml Falcon conical tube (Fisher Scientific, Pittsburgh) following the protocol described by Thiessen et al. (2016). To develop a standard curve for conidia quantification and primer sensitivity testing, sterile pairs of sampling rods were contaminated with known quantities of conidia, as described by Thiessen et al. (2016), with minor modifications. Conidia of the M. oryzae perennial ryegrass pathotype isolate FPH2014-07 (Columbus, $\mathrm{OH}$ ) were collected from 7-day-old cultures grown on oatmeal agar (Becton, Dickinson and Company, Franklin Lakes, NJ) by suspending them in $0.05 \%$ Tween 20 (Sigma-Aldrich, St. Louis) solution in nuclease-free water (Sigma-Aldrich). The concentration of the conidia suspension was estimated using a hemocytometer, and different volumes of the suspension or its dilutions were pipetted onto separate sampling rod pairs to a final concentration of approximately $25,50,100,1 \times 10^{3}, 1 \times 10^{4}$, or $1 \times 10^{5}$ conidia per pair of rods. Moreover, 10-conidia concentrations were obtained by manually transferring conidia to coated stainless steel rods using an eyelash brush. Rods were then air dried in a sterile laminar flow hood and stored at $4{ }^{\circ} \mathrm{C}$ until processing. A preliminary test made with four concentrations of conidia from infected grass tissue $(25,50,100$, and $1 \times 10^{3}$ conidia/rod) confirmed the accuracy of a standard curve made with the same conidia amounts collected from cultures grown on oatmeal agar (data not shown).

DNA extraction. Two different extraction protocols were used for DNA from pure fungal cultures for initial development of the qLAMP assay and for analysis of lab-prepared as well as fieldcollected sampling rods. DNA for the qLAMP assay development was extracted from 10-day-old fungal cultures grown on potato dextrose agar (Becton, Dickinson and Company) using the MoBio Power soil DNA Extraction kit (MoBio Laboratories, Carlsbad, CA) as directed by the manufacturer. Presence of DNA in each sample was verified prior to LAMP testing by gel electrophoresis, and approximate DNA concentrations were determined using a
NanoDrop 1000 spectrophotometer at $260 \mathrm{~nm}$ (NanoDrop Technologies, Wilmington, DE).

DNA from sampling rods was extracted using a Chelex protocol (Barkway et al. 2011), with modifications. Rods were aseptically transferred to sterile 2-ml self-standing screw-cap tubes (Axygen Scientific, Union City, CA) containing $200 \mu \mathrm{l}$ of 5\% Chelex 100 sodium form (Sigma-Aldrich) solution in nuclease-free water. Tubes were vortexed at maximum speed for $10 \mathrm{~s}$, immersed in boiling water for $5 \mathrm{~min}$, vortexed again for $10 \mathrm{~s}$, and boiled for an additional $5 \mathrm{~min}$. Tubes were then cooled down at room temperature for $5 \mathrm{~min}$ before centrifuging them at $14,100 \times g$ for $2 \mathrm{~min}$. Rods were aseptically removed from the extraction tubes and discarded, while the tubes were stored at $-20^{\circ} \mathrm{C}$ and the supernatant was used as template for LAMP reactions, which occurred no longer than 2 months after extraction. Each tube was subjected to only one cycle of freezing and thawing before use.

qLAMP assay development. LAMP primers and the fluorescent assimilating probe specific for the $M$. oryzae perennial ryegrass pathotype were designed on a genomic DNA region unique for $M$. oryzae strains isolated from $L$. perenne. To identify unique target regions, a Perl script was used to split the assembled genomic sequence of M. oryzae perennial ryegrass pathotype LPKY97-1 into 500-bp fragments with a 250-bp overlapping sequence (Korf et al. 2003). To identify candidate loci common to the perennial ryegrass pathotype, a BLASTn (Altschul et al. 1990) search was performed to align the segmented LPKY-97-1 genome to the genomic sequence of $M$. oryzae perennial ryegrass pathotype isolate FH. The LPKY97-1 sequence fragments without significant similarity to the perennial ryegrass pathotype isolate $\mathrm{FH}$ genomic sequence were removed. To identify candidate loci unique to the perennial ryegrass pathotype genome, a second BLASTn search was performed to align the LPKY97-1 sequences common to both perennial ryegrass pathotype isolates to a database containing the genomic sequences of $12 \mathrm{M}$. oryzae isolates collected from nine different host species. A final BLASTn search was performed to confirm that those candidate loci were present in both M. oryzae perennial ryegrass pathotype isolates. All BLASTn analyses were performed using default parameters. The list of $M$. oryzae isolates whose genomic sequences were used in the analysis is reported in Supplementary Table S1. LAMP primers were designed using PrimerExplorer (v. 4.0; Eiken Chemical Co., Tokyo) according to the specifications provided by Notomi et al. (2000), and the fluorescent assimilating probe was designed against the backward loop of the amplicon, as described by Kubota et al. (2011). The assimilating probe primes additional polymerization reactions starting from the backward loop region of the amplicon. During subsequent polymerization reactions, the fluorescent strand of the probe is incorporated into the amplicon, while the partially overlapping quench strand is displaced, resulting in an increase of fluorescence (Kubota et al. 2011). Primers and probe were synthesized by Eurofins MWG Operon (Eurofins MWG Operon, Huntsville, AL) and the sequences are reported in Table 1.

qLAMP reactions were performed in a final volume of $25 \mu \mathrm{l}$ containing $15 \mu \mathrm{l}$ of $1 \times$ no-dye Isothermal Master Mix (OptiGene, Horsham, UK), $2.8 \mu \mathrm{M}$ each internal primer (GLS2 FIP and GLS2

Table 1. Primers and probe used for the detection of Magnaporthe oryzae perennial ryegrass pathotype

\begin{tabular}{ll}
\hline Primer, probe & \multicolumn{1}{c}{ Sequence $\left(\mathbf{5}^{\prime} \mathbf{-} \mathbf{3}^{\prime}\right)^{\mathbf{a}}$} \\
\hline LAMP primer set & TGGT GCGG ATGC TAAT CGA \\
GLS2 F3 & CGCA AACA TCCA ATTG CTCA \\
GLS2 B3 & GAAC AGAC CGAT AGGC GGCA TAAG CCGA CGTT GATC TGC \\
GLS2 FIP & TCTG CGGA TTGT CGGA CTAC GACC GAAT AGCC ACAT CTCA CG \\
GLS2 BIP & \\
Assimilating probe & FAM-ACGC TGAG GACC CGGA TGCG AATG CGGA TGCG GATG CCGA TGCA GAAT GCGT TCCA CG \\
GLS2, fluorescent strand & TCGG CATC CGCA TCCG CATT CGCA TCCG GGTC CTCA GCGT-BHQ \\
Quench strand & \\
\hline a FAM = 6-carboxyfluorescein, underlined fragment of the fluorescent strand of the assimilating probe acts as backward loop primer, and BHQ = Black Hole \\
Quencer-1 (Biosearch Technologies, Novato, CA) (Kubota et al. 2011). \\
b Assimilating probe was designed as for Kubota et al. (2011).
\end{tabular}


BIP), $0.28 \mu \mathrm{M}$ each external primer (GLS2 F3 and GLS2 B3), $0.092 \mu \mathrm{M}$ assimilating probe fluorescent strand, $0.184 \mu \mathrm{M}$ quencher strand, and $5 \mu \mathrm{l}$ of template DNA. Real-time qLAMP reactions were carried out in 0.2-ml, eight-tube strips on a CFX96 Touch Real-Time PCR Detection System (Bio-Rad Laboratories, Hercules, CA), and were held at $66^{\circ} \mathrm{C}$ for 50 cycles of $1 \mathrm{~min}$ each, followed by $5 \mathrm{~min}$ at $80^{\circ} \mathrm{C}$ to inactivate the polymerase. Data acquisition and cycle threshold $\left(\mathrm{C}_{\mathrm{t}}\right.$, corresponding here to minute threshold, because every cycle was $1 \mathrm{~min}$ long) analysis were performed using Bio-Rad CFX Manager 3.1 software (Bio-Rad Laboratories), using the automatic baseline set by the software and a manually set baseline threshold of 110 .

Specificity of the qLAMP assay was tested using DNA extracted from cultures of the fungal isolates reported in Table 2, carrying out each reaction in duplicate. Isolates were chosen either because they represented different pathotypes of $M$. oryzae or for their common occurrence on perennial ryegrass. Because it was not possible for our laboratory to directly test $M$. oryzae strains isolated from wheat due to quarantine issues, an in silico specificity test of the target region was performed using BLASTn against the genome of M. oryzae isolate PY86 from wheat (Castroagudín et al. 2015), which is the isolate most closely related, phylogenetically, to the L. perenne clade (M. Farman, personal communication). The analysis was performed by Dr. M. Farman, Department of Plant Pathology, University of Kentucky.

Sensitivity of the assay was tested against a standard curve of conidial spore dilutions on sampling rods prepared as described above. Six independent extractions of each spore concentration were examined, carrying out each reaction in triplicate. The standard curve was then generated by averaging the $C_{t}$ value of each spore concentration from the six independent DNA extractions. All qLAMP reactions carried out during the assay development phase contained two template-free negative controls, and one reaction with $50 \mathrm{ng}$ of DNA extracted from the mycelium of isolate FPH2014-07 as positive control.

Detection and quantification of airborne inoculum in turfgrass fields. Custom impaction spore traps (Fig. 1), modified from Thiessen et al. (2016) and similar to the Rotorod Sampler (Sampling Technologies Inc., Minnetonka, MN), were placed in two L. perenne 15-by-18-m field plots (plot A and plot B) established at the Ohio Turfgrass Foundation Research and Education Facility in Columbus in July 2015 (Fig. 2). GLS infection had never been observed at the facility prior to inoculation of the experimental plots. Plots were $30 \mathrm{~m}$ apart from each other along the north-south line, and were further divided into 25 subplots by

Table 2. Fungal isolates used to test the specificity of the Magnaporthe oryzae perennial ryegrass pathotype quantitative loop-mediated isothermal amplification assay

\begin{tabular}{|c|c|c|c|c|c|c|}
\hline Isolate & Pathogen species & Original host & Locality & Year of isolation & Reference or collector & Amplification result \\
\hline FPH2014-07 & Magnaporthe oryzae & Lolium perenne & Ohio & 2014 & C. Villari & + \\
\hline FPH2015-43 & M. oryzae & L. perenne & Ohio & 2015 & C. Villari & + \\
\hline FPH2015-45 & M. oryzae & L. perenne & Ohio & 2015 & C. Villari & + \\
\hline FPH2015-46 & M. oryzae & L. perenne & Ohio & 2015 & C. Villari & + \\
\hline FPH2015-47 & M. oryzae & L. perenne & Ohio & 2015 & C. Villari & + \\
\hline FPH2015-176 & M. oryzae & L. perenne & Ohio & 2015 & C. Farinas & + \\
\hline PgPA33B- $01^{a}$ & M. oryzae & L. perenne & Pennsylvania & 1999 & W. Uddin & + \\
\hline PgPA33B-06a & M. oryzae & L. perenne & Pennsylvania & 1999 & W. Uddin & + \\
\hline PgPA05T-01 & M. oryzae & L. perenne & Pennsylvania & 1998 & W. Uddin & + \\
\hline PgPA $35 \mathrm{H}-4.1^{\mathrm{a}}$ & M. oryzae & L. perenne & Pennsylvania & 2000 & W. Uddin & + \\
\hline PgPA18C-04a & M. oryzae & L. perenne & Pennsylvania & 1998 & W. Uddin & + \\
\hline LpKY-97-1 & M. oryzae & L. perenne & Kentucky & 1997 & M. Farman & + \\
\hline LpKY $-96^{b}$ & M. oryzae & L. perenne & Kentucky & 1996 & P. Vincelli & + \\
\hline GG-98c & M. oryzae & L. perenne & Kentucky & 1998 & P. Vincelli & + \\
\hline GG-5-4bc & M. oryzae & L. perenne & Kentucky & 2001 & P. Vincelli & + \\
\hline GG $3 \mathrm{H}-4-2^{\mathrm{d}}$ & M. oryzae & L. perenne & Kentucky & 2001 & P. Vincelli & + \\
\hline GG-14-3-9 & M. oryzae & L. perenne & Kentucky & 2001 & P. Vincelli & + \\
\hline GG-14-2-2 & M. oryzae & L. perenne & Kentucky & 2001 & P. Vincelli & + \\
\hline PL1-1 ${ }^{\mathrm{e}}$ & M. oryzae & L. multiflorum & Kentucky & 2003 & P. Vincelli & - \\
\hline PL2-1 ${ }^{\mathrm{e}}$ & M. oryzae & L. multiflorum & Kentucky & 2003 & P. Vincelli & + \\
\hline PL3-1e & M. oryzae & L. multiflorum & Kentucky & 2003 & P. Vincelli & - \\
\hline S-4 & M. oryzae & Setaria viridis & Maryland & 2012 & G. Peterson & - \\
\hline $\mathrm{SSOH}^{\mathrm{f}}$ & M. oryzae & Stenotaphrum secundatum & Florida & - & M. Boehm & - \\
\hline CHE86056ZB13g & M. oryzae & Oryza sativa & China & - & G.-L. Wang & - \\
\hline BF 19 & M. oryzae & O. sativa & Burkina Faso & 2013 & BBSRC $^{\mathrm{h}}$ & - \\
\hline $\mathrm{BF} 9$ & M. oryzae & O. sativa & Burkina Faso & 2013 & BBSRC & - \\
\hline BF 36 & M. oryzae & O. sativa & Burkina Faso & 2013 & BBSRC & - \\
\hline KE 10 & M. oryzae & O. sativa & Kenya & 2013 & BBSRC & - \\
\hline KE 16 & M. oryzae & O. sativa & Kenya & 2013 & BBSRC & - \\
\hline KE 21 & M. oryzae & O. sativa & Kenya & 2013 & BBSRC & - \\
\hline UG 04 & M. oryzae & O. sativa & Uganda & - & BBSRC & - \\
\hline TZ 08 & M. oryzae & O. sativa & Tanzania & 2013 & BBSRC & - \\
\hline FPH2014-10 & Leptosphaerulina trifolii & L. perenne & Ohio & 2014 & C. Villari & - \\
\hline FPH2014-11 & Curvularia sp. & L. perenne & Ohio & 2014 & C. Villari & - \\
\hline \multicolumn{7}{|c|}{$\begin{array}{l}\text { a Tosa et al. } 2007 . \\
\text { ' Farman et al. } 2002 . \\
\text { c Vincelli and Dixon } 2002 . \\
\text { d Kim et al. } 2003 . \\
\text { e Vincelli et al. } 2008 . \\
\text { f Jo et al. } 2007 . \\
\text { g Liu et al. } 2002 \text {. } \\
\text { " } \text { BBSRC = Biotechnology and Biological Sciences Research Council. The isolates are a subset of the "African Core" generated as part of the "Durable rice blast } \\
\text { resistance through genomic analysis of the host-pathogen interaction, BB/J012157/1" and stored in the biobank culture storage facility at ILRI/BecA hub in }\end{array}$} \\
\hline
\end{tabular}


a 3-by-3.6-m grid. Plots were watered once a day for $7 \mathrm{~min}$ throughout the duration of the experiment, and mowed to $7.6 \mathrm{~cm}$ every other week. Daily weather data were recorded through the Ohio Agricultural Research and Development Center weather system, Columbus unit (series 2000; WatchDog Spectrum Technology Inc., Plainfield, IL), which is located $50 \mathrm{~m}$ away from plot B.

In total, four traps were placed in the subplots corresponding to the external corners of each plot (Fig. 2). Traps were placed so that the sampling arm was within $15 \mathrm{~cm}$ from ground level. Each trap was calibrated to sample air at $59.5 \pm 1$ liter $\mathrm{min}^{-1}$ by spinning two sampling rods at approximately $1 \mathrm{~m} \mathrm{~s}^{-1}$. Before inoculation of the plots, two traps in plot A were run for $72 \mathrm{~h}$ to collect air biota samples and test primer specificity against large quantities of background DNA.

In order to ensure an infection event and minimize the effect of adverse weather conditions on the outcome of this study, plots A and B were inoculated on two separate days 1 week apart from each other, on 24 and 31 August 2015, respectively. Inoculum was prepared as described by Harmon and Latin (2005). Briefly, perennial ryegrass was grown for 3 weeks in the greenhouse in six plastic flats ( 30 by $61 \mathrm{~cm}$ ) filled with Metro-Mix 360 growing medium (Scotts-Sierra Horticultural Products Co., Marysville, OH). Flats were then inoculated with a conidial suspension of $10^{5}$ spores $\mathrm{ml}^{-1}$ collected from 7-day-old cultures of $M$. oryzae isolate FPH2014-07 grown on oatmeal agar, and incubated for $96 \mathrm{~h}$ in a growth chamber at $28^{\circ} \mathrm{C}$, $98 \%$ relative humidity, and an 18 -h photoperiod. After the incubation period, flats were returned to the greenhouse and maintained at a temperature of 25 to $30^{\circ} \mathrm{C}$ for 1 week to allow symptom development. GLS-symptomatic grass was cut at the crown and allowed to air dry inside paper bags placed in a chemical fume hood for 25 days. Thoroughly mixed dry clippings were dispersed over five subplots in each experimental plot (Fig. 2). Inoculation occurred at sunset, and inoculated subplots were immediately covered with plastic tarp to ensure maintenance of an adequate temperature and relative humidity during the first night. The tarp was removed within an hour from sunrise the following morning.

All traps within each plot were run continuously, starting from the morning after inoculation and until appearance of visible symptoms within the corresponding subplot. To monitor for symptom emergence, each subplot was thoroughly visually inspected on a daily basis by examining individual leaf blades for lesions for a total of 23 days after inoculation. Disease incidence was estimated as the percentage of subplots where at least one individual lesion was found. The first symptomatic blades from each plot were destructively sampled to assess the presence of $M$. oryzae spores under a dissecting scope ( $\times 8$ magnification) after a 12 -h incubation in a moist chamber.

Sampling rods were replaced daily starting $24 \mathrm{~h}$ after the activation of the traps and transported to the laboratory in 15-ml Falcon conical tubes. Throughout the duration of the experiment, if rods detached from the traps, likely due to coming into contact with an animal or in the few cases of trap malfunctioning, data points were considered as missing data.

DNA extraction from field-collected sampling rods and qLAMP reactions were performed as described above. A set of sampling rods contaminated with approximately 5,000 conidia of the isolate FPH2014-07 was included in each set of DNA extractions as a positive control for extraction efficiency (Thiessen et al. 2016). All qLAMP reactions were carried out in triplicate, and every reaction plate (made by assembling strips) contained the positive control for extraction efficiency, $1 \times 10^{4}$ and $1 \times 10^{5}$ conidia positive controls for reaction efficiency, and template-free negative controls. Each field-collected sample was considered positive if at least two of the three replicates had a positive response, and conidia quantification was determined by comparing the average $C_{t}$ value to the assay standard curve described above.

\section{Results}

Specificity and sensitivity of qLAMP assay. The qLAMP assay developed in this study was specific to $M$. oryzae strains isolated

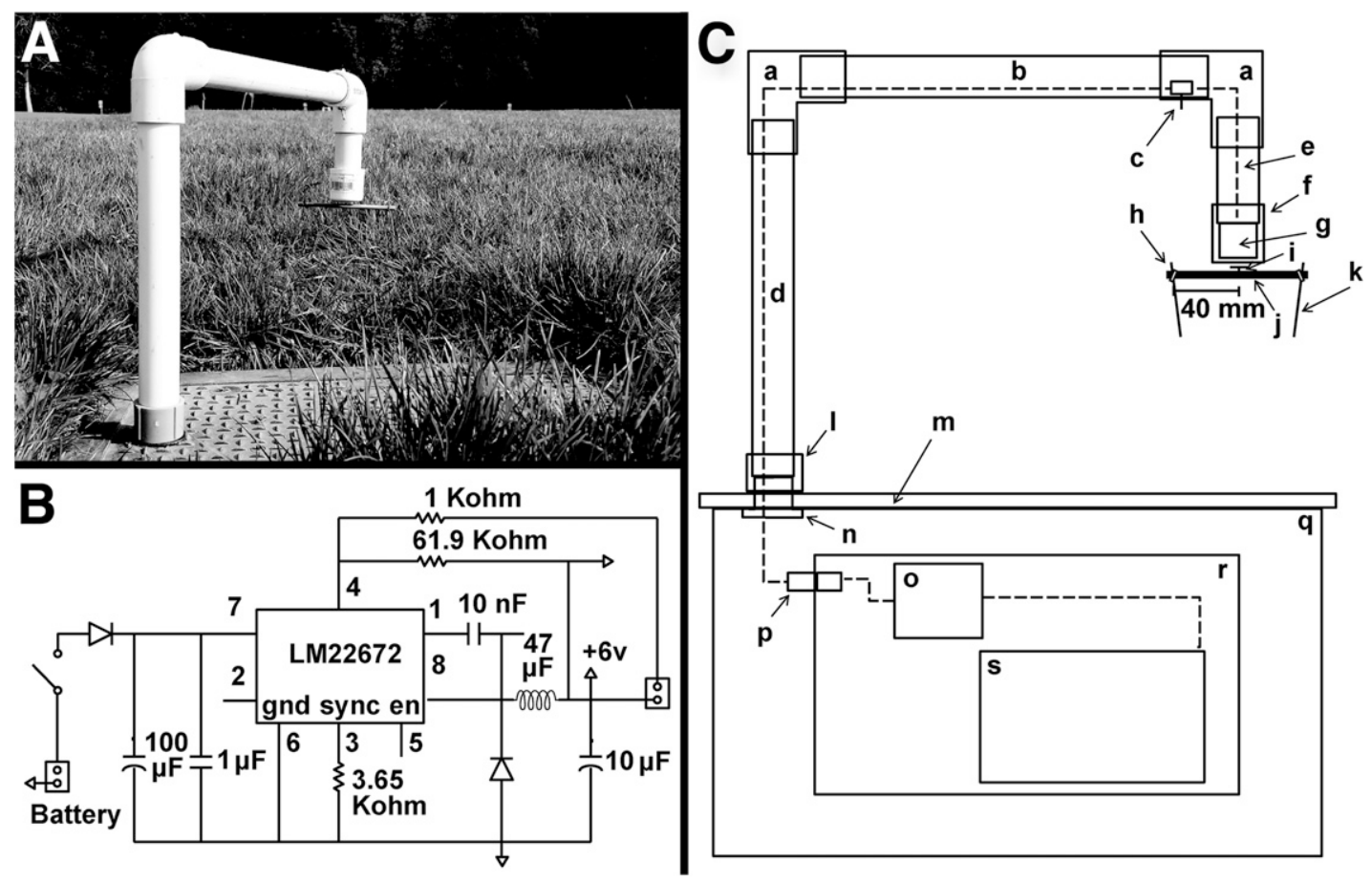

Fig. 1. Impaction spore trap design as used in the study. A, Trap placed in the turfgrass field to catch Magnaporthe oryzae spores. Valve box is buried in the ground. Sampling arm can be rotated to a trapping configuration (i.e., with the sampling rods located above the perennial ryegrass blades) or to a mowing configuration (i.e., with the sampling rods located above the valve box to facilitate mowing operations). B, Circuit diagram of the voltage regulator used to control motor speed. C, Spore trap components: (a) 19-mm PVC elbow; (b) 19-mm internal diameter (ID) by 355-mm segment of PVC pipe; (c) toggle switch; (d) 19-mm ID by 254-mm segment of PVC pipe; (e) 19-mm ID by 65 -mm segment of PVC pipe; (f) 19-mm PVC end cap; (g) Nichibo DC-130SB-10410R DC motor (Nichibo, Taipei City, Taiwan); (h) silicone o-ring (4.5-mm ID); (i) SuGru moldable silicon (FormFormForm, London) placed around motor spindle in a cone for water proofing; (j) 4.7-by-90-mm aluminum sampling arm with 40-mm sampling radius; (k) 1.1-by-40-mm stainless steel rods; (I) 19-mm male PVC terminal adapter; ( $\mathrm{m}$ ) irrigation value cover box (356 by 482 by $320 \mathrm{~mm}$ ); (n) 19-mm lock nut; (p) Con-X 2-pin cable pin and panel mount circular weather tight connector (Conxall, Villa Park, IL); (o) circuit board in panel B; (r) U.S. military 50 BMG caliber ammo box; and (s) 12-V, 9-Ah sealed lead acid battery. Dashed lines are Carol Brand 20-gauge two-strand PVC jacketed multiconductor electrical cable (General Cable, Highland Heights, KY). 
from $L$. perenne, producing a positive response for all but only the tested target strains (Table 2). In only one case, $M$. oryzae isolate PL2-1 from L. multiflorum (Vincelli et al. 2008) gave a positive response. The in silico specificity test of the target region against the genome of $M$. oryzae isolate PY86 gave zero hits as a result.

The qLAMP primers were able to positively amplify DNA extracted from $10,25,50,100,1 \times 10^{3}, 1 \times 10^{4}$, and $1 \times 10^{5}$ conidia within $45 \mathrm{~min}$, showing high primer sensitivity. The standard curve generated from the sampling rods coated with known quantities of conidia had a quadratic response (Fig. 3) due to amplicon concatenation occurring during the reaction (Mori et al. 2001). Even though DNA corresponding to 10 conidia was successfully amplified (average $C_{t} \pm$ standard error $=39.48 \pm 2.18$ ), only $50 \%$ of extractions, and not all replicates within an extraction, consistently produced a positive result. Moreover, the coefficient of determination of the standard curve was sensibly lower $\left(R^{2}=0.922\right)$ when including the 10 -conidia point, even though the coefficient of variation $(\mathrm{CV})$ for the point was $12.340 \%$. This error is still within the recommended threshold level of 20\% (Clinical Laboratory and Standards Institute 2004; Holstein et al. 2015; Hospodsky et al. 2010). For this reason, we opted for a lower range of quantification equal to 25 conidia, which corresponds to the lower point of the curve that consistently produced a positive reaction. During field samples analysis, all positive results below this threshold were considered as confirmed presence of conidial spores but the amount was not quantified. Excluding the 10-conidia point, the $\mathrm{CV}$ for all other points of the curve was below $6 \%$.

Detection and quantification of airborne inoculum in turfgrass fields. Air biota samples collected before plot inoculation did not produce a positive amplification when tested with the qLAMP primers, suggesting that GLS was not occurring in the area before the beginning of the experiment, and that the primers are specific even in the presence of large quantities of background DNA.
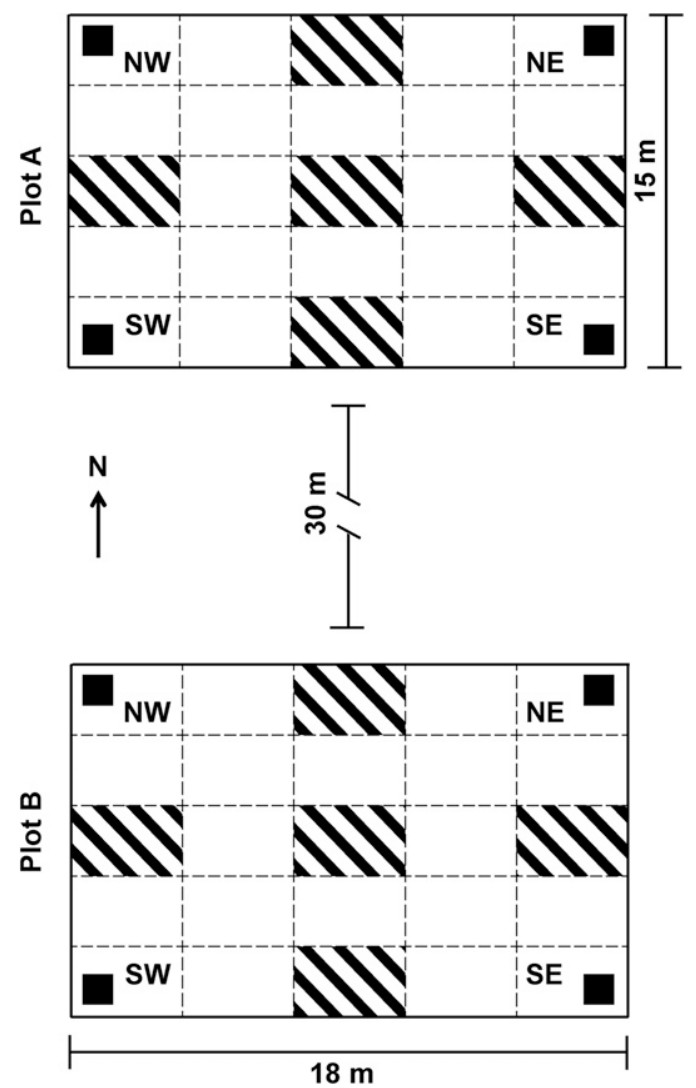

Fig. 2. Schematic representation of the two experimental plots established at the Ohio Turfgrass Foundation Research and Education Facility, Columbus. Each plot was divided into 25 subplots. Black squares $=$ impaction spore traps and dashed sectors $=$ subplots inoculated with Magnaporthe oryzae. Abbreviations: NW, NE, SW, and SE = northwest, northeast, southwest, and southeast, respectively.
The first visible symptoms of GLS developed 15 and 8 days after inoculation in plot A and B, respectively. The typical disease symptoms of a blue-gray appearance of the grass that is thinned by dead, decaying leaf blades were not observed in any subplot during the course of the experiment. The symptoms observed were lesions on individual leaves. After the first lesions appeared, the disease quickly spread in the two plots but was still only detectable by thorough examination of leaf blades. Within 23 days postinoculation, 56 and $84 \%$ of subplots in plot A and B, respectively, had at least one lesion. $M$. oryzae airborne inoculum was detected in all eight traps before the appearance of visible symptoms within their corresponding subplot, and seven of eight traps were able to detect inoculum up to 12 days before the appearance of visible symptoms within the whole plot (Fig. 4). In the northeast trap subplot of plot B, symptoms never developed throughout the observation period but the corresponding trap was still able to detect inoculum (Fig. 4).

\section{Discussion}

In this work, we described the development of a qLAMP assay coupled with a spore trap system for the rapid detection and quantification of airborne inoculum of the $M$. oryzae perennial ryegrass pathotype, the causal agent of GLS, and the testing of its suitability for implementation in turfgrass fields. The qLAMP assay-trap system was found to be sensitive and specific to $M$. oryzae perennial ryegrass isolates, with the exception of one case. It was capable of detecting and quantifying airborne inoculum in the field up to 12 days before the appearance of visible symptoms, at a distance of approximately $6 \mathrm{~m}$ from the closest potential source of inoculum. The number of spores detected by the qLAMP assay-trap system was comparable with the number of spores reported to be associated with the beginning of an epidemic phase (Harmon and Latin 2005).

The only nonspecific amplification of the qLAMP assay was with M. oryzae isolate PL2-1 from L. multiflorum (Vincelli et al. 2008). However, recent phylogenetic analyses showed that this strain is the only one of the three isolates from L. multiflorum belonging to the L. perenne clade (M. Farman, personal communication). This result suggests that, despite its original isolation from a different host, it might actually belong to the perennial ryegrass pathotype. Indeed, isolates from $L$. perenne are able to cross-infect other hosts (Viji et al. 2001). For this reason, the qLAMP assay developed in this study is still likely to be specific to $M$. oryzae perennial ryegrass pathotype. However, it also indicates that, during further development of the assay, the potential of cross reaction needs to be continuously assessed.

The qLAMP assay was capable of amplifying in less than 45 min as few as 10 conidia, with a lower range of quantification equal to 25 conidia. The lower sensitivity of this assay compared with other LAMP assays designed against the internal transcribed spacer (ITS) region of the ribosomal RNA (Thiessen et al. 2016) is probably

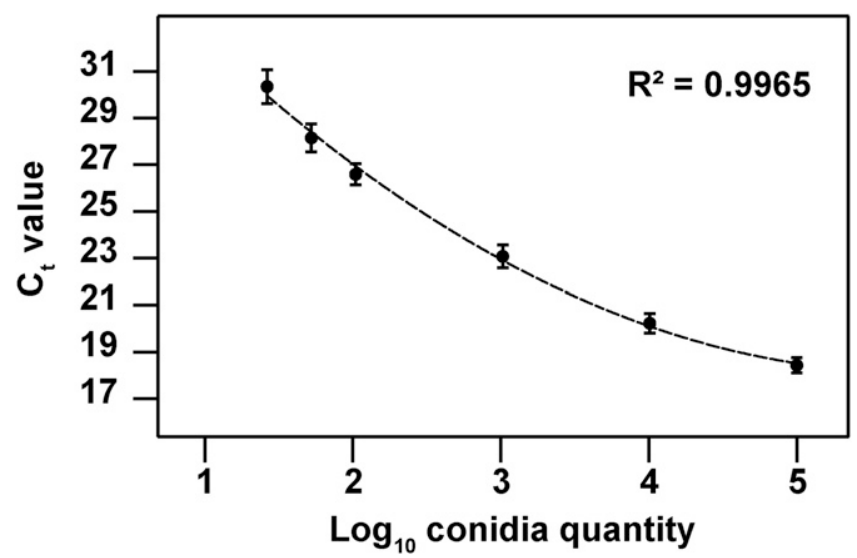

Fig. 3. Standard curve for Magnaporthe oryzae conidia quantification with the $M$. oryzae perennial ryegrass pathotype assimilating probe-based loop-mediated isothermal amplification assay. Each point corresponds to the average of six independent extractions ( \pm standard error). Cycle threshold $\left(\mathrm{C}_{t}\right)$ corresponds to minute threshold, because every cycle was 1 min long. 
due to the lower copy number of the genomic DNA region used in this study (one copy) as opposed to the ITS region (Bruns et al. 1991; Liew et al. 1998). Because mature M. oryzae conidia are three celled and have three haploid nuclei (Caracuel-Rios and Talbot 2007), in the unlikely event that all copies of the target region end up in the reaction, a maximum of three copies of the target region per conidium is expected, which is below the nine copies per reaction necessary to initiate the qLAMP reaction (Kubota et al. 2011). At any rate, 10 conidia trapped in a day despite a sampling capability of air at 60 liters $\min ^{-1}$ is still an extremely low number compared with the aerial concentration of $M$. oryzae conidia considered to be infectious (Harmon and Latin 2005; Pinnschmidt et al. 1993). Variability was observed in the number of conidia collected by each trap in a given day, even among traps that were in close proximity to inoculated subplots. This variability is likely due to the stochasticity of air turbulence, velocity, and direction in relation to the inoculum sources and the traps and how they affected dispersion (Mahaffee and Stoll 2016). There were likely very specific events that had enough energy for spores to escape the matrix they where sporulating on and disperse on the prevailing air currents (Gleicher et al. 2014). In addition to being highly sensitive, the developed assay was also fairly precise. In fact, the $\mathrm{CV}$ of the standard curve built to quantify conidia was below $6 \%$ for all curve points, differing only a little from the precision of classic real-time PCR, which has an expected CV below $2 \%$ (Klein 2002). Moreover, at the cost of a little loss in precision, qLAMP technology comes with the great advantage of rapidity (Kubota et al. 2011; Notomi et al. 2000) and the possibility to be field implemented (Jenkins et al. 2011; Mahaffee 2014). For the assay developed in this study, results could be obtained in less than 90 min, including the time devoted to DNA extraction.

LAMP is a robust technique (Jenkins et al. 2014; Tomlinson et al. 2007,2013 ) and, in the case of grape powdery mildew, it was shown to be suitable for implementation by growers to initiate and time fungicide applications upon inoculum detection using a nonquantitative, turbidity-based LAMP assay (Thiessen et al. 2016). Following Thiessen et al. (2016) and Kubota et al. (2011), we developed a qLAMP assay-trap system that could be implemented in field settings with the use of portable devices capable of reading fluorescence, such as SmartDART (Diagenetix Inc. Honolulu, HI). The Smart-DART has been recently shown to be highly efficient for detecting bacterial pathogens (Jenkins et al. 2014) and could prove useful for turf managers.

The field implementation of the qLAMP assay-trap system developed in this study has the potential to allow for an accurate and timely detection of GLS spores before development of visible symptoms,
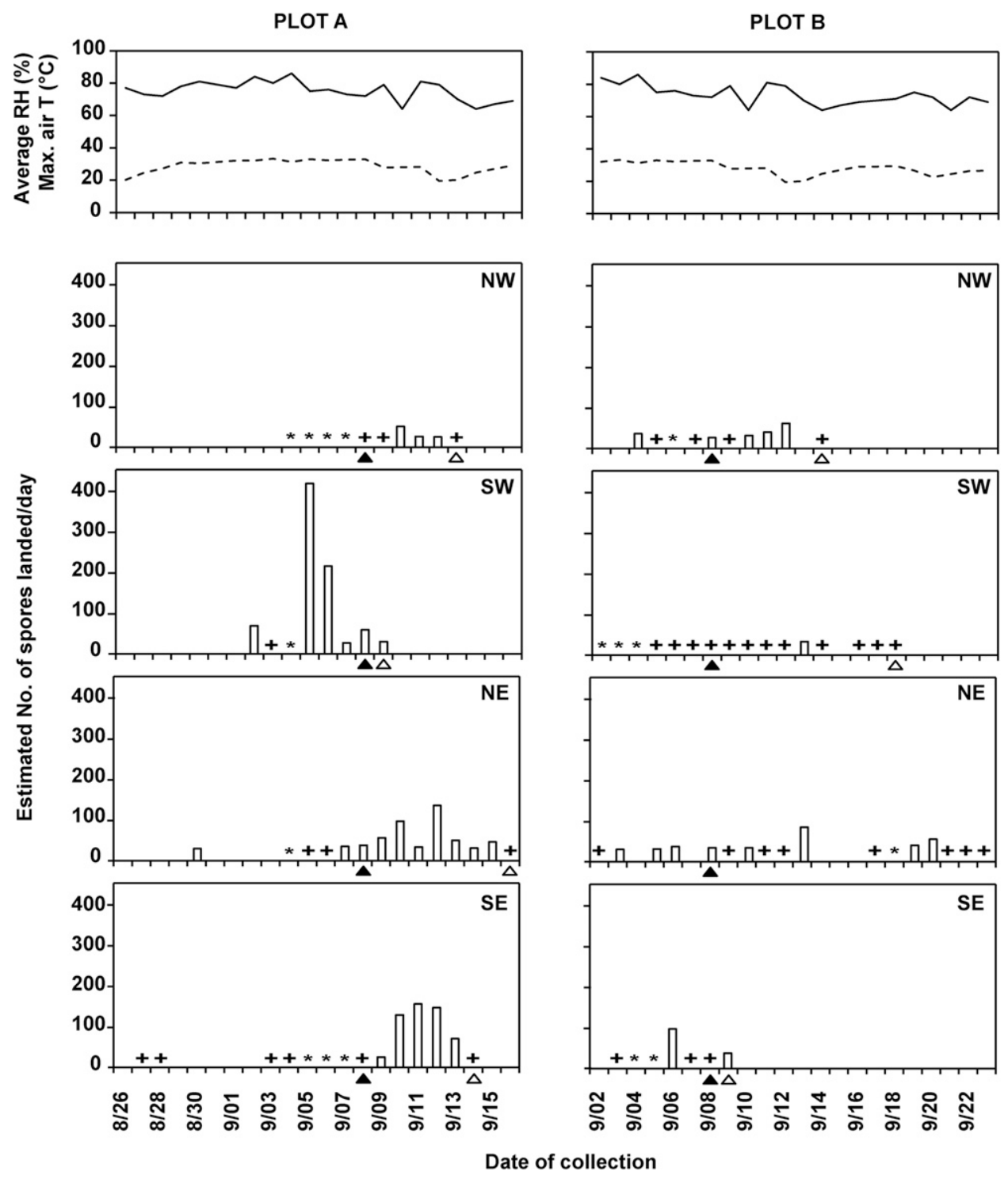

Fig. 4. Estimated number of Magnaporthe oryzae conidia landed per day on each subplot impaction spore trap. Each trap was run continuously, starting from the morning after inoculation and until emergence of symptoms within the corresponding subplot (open triangles). Symptoms in the plot emerged on 8 September (closed triangles). Plus symbols $(+)=$ positive detection below limit of quantification and asterisks $\left(^{*}\right)=$ missing data. Weather data are reported in the two top charts. Dashed line $=$ daily maximum air temperature and solid line $=$ daily average relative humidity. 
which is particularly useful for pathogens such as $M$. oryzae that incite sporadic disease outbreaks or may be easily confused with less damaging pathogens at the beginning of the disease epidemic (Harmon et al. 2003). After field inoculation, symptoms were first detected on the same day in both plots, despite the two plots being inoculated 1 week apart from each other and daily scouting for disease. This was most likely due to different environmental conditions during the study period. During the first week of the experiment, when only plot A was inoculated, temperatures were below seasonal average and not favorable to $M$. oryzae sporulation and GLS disease development (Henry and Andersen 1948; Uddin et al. 2003b,c). Daily maximum temperatures raised above $30^{\circ} \mathrm{C}$ and daily average relative humidity reached $80 \%$ only during the second week of the study, a few days after inoculation of plot B; and, as expected, first symptoms developed within a week of favorable weather conditions (Harmon and Latin 2005). Field implementation of the qLAMP assay would significantly improve IPM for GLS because growers, managers, and turf superintendents would be able to rapidly detect the presence of the causal agent and determine the appropriate actions prior to the first effort to control, which has been shown to reduce fungicide use in other pathosystems (Thiessen et al. 2016). Detecting and quantifying airborne inoculum has been used extensively to guide disease management decisions; however, there are several epidemiological considerations to bear in mind in order to not over or underestimate the severity of the epidemic (Hardwick 2006; Mahaffee 2014; West et al. 2008). Development of the assay and testing of its suitability for implementation in artificially inoculated turfgrass fields are only the first steps toward the use of the assay as a disease management decision support tool. Future research will build upon this work and will include testing the suitability of the assay to detect and quantify pathogen inoculum in naturally infected fields, with the purpose of examining the correlation between the airborne spore concentrations as determined by the LAMP assay, the observed disease levels in the field, environmental parameters, and turf management factors. The information generated should allow the identification of thresholds of spore concentrations at which disease occurs. Based on the identified thresholds, a management plan guided by the spore trap-LAMP system would be compared with a grower standard plan to assess which one confers the best level of disease control with a lower number of fungicide applications. A second phase would also include training of practitioners and personnel in implementing the qLAMP assay-trap system and the Smart-DART or other portable devices. There will also be further specificity testing during the continued development and implementation.

In summary, this study represents only the proof of concept for the suitability of qLAMP technology application to turfgrass ecosystems. As an effort toward the increased implementation of IPM in turfgrass culture, future research will build upon this study to ultimately provide turf managers and superintendents with decision support tools that they could implement for a more sustainable management of fungal diseases of turfgrass.

\section{Acknowledgments}

This work was supported by The Ohio Agricultural Research and Development Center SEEDS Grants Program award number 2014-021, the United States Department of Agriculture (USDA) National Institute of Food and Agriculture (NIFA) Hatch project number 1004939, and USDA Agricultural Research Service (ARS) CRIS 5358-22000-039-OOD. This project has been funded, in part, by Agriculture and Food Research Initiative Competitive Grant number 2013-68004-20378 (Blast Integrated Project) from the USDA NIFA. We thank M. Farman (University of Kentucky) for providing some of the genomic sequences of $M$. oryzae isolates used in this study and for his support with in silico analysis; P. Vincelli (University of Kentucky), G. Peterson (USDA-ARS), G.-L. Wang, and M. Boehm (The Ohio State University) and the BBSRC BB/J012157/1 for sharing some of the M. oryzae isolates used in this study; J. W. Rimelspach, T. Hicks and C. Farinas (The Ohio State University), and L. D. Thiessen (Oregon State University) for technical assistance; and two anonymous reviewers for their valuable suggestions to improve the manuscript.

\section{Literature Cited}

Altschul, S. F., Gish, W., Miller, W., Myers, E. W., and Lipman, D. J. 1990. Basic local alignment search tool. J. Mol. Biol. 215:403-410.
Barkway, C. P., Pocock, R. L., Vrba, V., and Blake, D. P. 2011. Loop-mediated isothermal amplification (LAMP) assays for the species-specific detection of Eimeria that infect chickens. BMC Vet. Res. 7:67.

Bruns, T. D., White, T. J., and Taylor, J. W. 1991. Fungal molecular systematics. Annu. Rev. Ecol. Syst. 22:525-564.

Caracuel-Rios, Z., and Talbot, N. J. 2007. Cellular differentiation and host invasion by the rice blast fungus Magnaporthe grisea. Curr. Opin. Microbiol. 10:339-345.

Castroagudín, V. L., Ceresini, P. C., de Oliveira, S. C., Reges, J. T. A., Maciel, J. L. N. Bonato, A. L. V., Dorigan, A. F., and McDonald, B. A. 2015. Resistance to QoI fungicides is widespread in Brazilian populations of the wheat blast pathogen Magnaporthe oryzae. Phytopathology 105:284-294.

Clinical Laboratory and Standards Institute. 2004. Protocols for determination of limits of detection and limits of quantitation, approved guidelines. CLSI document EP17. Clinical Laboratory and Standards Institute, Wayne, PA.

Couch, B. C., and Kohn, L. M. 2002. A multilocus gene genealogy concordant with host preference indicates segregation of a new species, Magnaporthe oryzae, from M. grisea. Mycologia 94:683-693.

Elkins, R. B., Temple, T. N., Shaffer, C. A., Ingels, C. A., Lindow, S. B., Zoller, B. G., and Johnson, K. B. 2015. Evaluation of dormant-stage inoculum sanitation as a component of a fire blight management program for fresh market Bartlett pear. Plant Dis. 99:1147-1152.

Farman, M. L. 2002. Pyricularia grisea isolates causing gray leaf spot on perennial ryegrass (Lolium perenne) in the United States: Relationship to P. grisea isolates from other host plants. Phytopathology 92:245-254.

Farman, M. L., Eto, Y., Nakao, T., Tosa, Y., Nakayashiki, H., Mayama, S., and Leong, S. A. 2002. Analysis of the structure of the AVR1-CO39 avirulence locus in virulent rice-infecting isolates of Magnaporthe grisea. Mol. PlantMicrobe Interact. 15:6-16

Gleicher, S. C., Chamecki, M., Isard, S. A., Pan, Y., and Katul, G. G. 2014 Interpreting three-dimensional spore concentration measurements and escape fraction in a crop canopy using a coupled Eulerian-Lagrangian stochastic model. Agric. For. Meteorol. 194:118-131.

Hardwick, N. V. 2006. Disease forecasting. Pages 239-267 in: The Epidemiology of Plant Diseases. B. M. Cooke, D. Gareth Jones, and B. Kaye, eds. Springer, Dordrecht, The Netherlands.

Harmon, P. F., Dunkle, L. D., and Latin, R. 2003. A rapid PCR-based method for the detection of Magnaporthe oryzae from infected perennial ryegrass. Plant Dis. 87:1072-1076.

Harmon, P. F., and Latin, R. 2005. Winter survival of the perennial ryegrass pathogen Magnaporthe oryzae in North Central Indiana. Plant Dis. 89:412-418.

Held, D. W., and Potter, D. A. 2012. Prospects for managing turfgrass pests with reduced chemical inputs. Annu. Rev. Entomol. 57:329-354.

Henry, B., and Andersen, A. 1948. Sporulation by Piricularia oryzae. Phytopathology $38: 265-278$

Holstein, C. A., Griffin, M., Hong, J., and Sampson, P. D. 2015. Statistical method for determining and comparing limits of detection of bioassays. Anal. Chem. 87:9795-9801.

Hospodsky, D., Yamamoto, N., and Peccia, J. 2010. Accuracy, precision, and method detection limits of quantitative PCR for airborne bacteria and fungi. Appl. Environ. Microbiol. 76:7004-7012.

Jenkins, D. M., Jones, J. B., and Kubota, R. 2014. Evaluation of portable DNAbased technologies for identification of Ralstonia solanacearum race 3 biovar 2 in the field. Biol. Eng. Trans. 7:83-96.

Jenkins, D. M., Kubota, R., Dong, J., Li, Y., and Higashiguchi, D. 2011. Handheld device for real-time, quantitative, LAMP-based detection of Salmonella enterica using assimilating probes. Biosens. Bioelectron. 30:255-260.

Jo, Y.-K., Wang, G.-L., and Boehm, M. J. 2007. Expression analysis of rice defenserelated genes in turfgrass in response to Magnaporthe grisea. Phytopathology 97:170-178.

Kim, Y.-S., Dixon, E. W., Vincelli, P., and Farman, M. L. 2003. Field resistance to strobilurin (QoI) fungicides in Pyricularia grisea caused by mutations in the mitochondrial cytochrome b gene. Phytopathology 93:891-900.

Klein, D. 2002. Quantification using real-time PCR technology: Applications and limitations. Trends Mol. Med. 8:257-260.

Kogovšek, P., Hodgetts, J., Hall, J., Prezelj, N., Nikolić, P., Mehle, N., Lenarčič, R., Rotter, A., Dickinson, M., Boonham, N., Dermastia, M., and Ravnikar, M. 2015. LAMP assay and rapid sample preparation method for on-site detection of flavescence dorée phytoplasma in grapevine. Plant Pathol. 64:286-296.

Korf, I., Yandell, M., and Bedell, J. 2003. Blast. O'Reilly \& Associates, Sebastopol, CA.

Kubota, R., Alvarez, A. M., Su, W. W., and Jenkins, D. M. 2011. FRET-based assimilating probe for sequence-specific real-time monitoring of loop-mediated isothermal amplification (LAMP). Biol. Eng. Trans. 4:81-100.

Lievens, B., and Thomma, B. P. H. J. 2005. Recent developments in pathogen detection arrays: Implications for fungal plant pathogens and use in practice. Phytopathology 95:1374-1380.

Liew, E. C. Y., Maclean, D. J., and Irwin, J. A. G. 1998. Specific PCR based detection of Phytophthora medicaginis using the intergenic spacer region of the ribosomal DNA. Mycol. Res. 102:73-80.

Liu, G., Lu, G., Zeng, L., and Wang, G.-L. 2002. Two broad-spectrum blast resistance genes, $\mathrm{Pi} 9(\mathrm{t})$ and $\mathrm{Pi} 2(\mathrm{t})$, are physically linked on rice chromosome 6. Mol. Genet. Genomics 267:472-480. 
Mahaffee, W. F. 2014. Use of airborne inoculum detection for disease management decisions. Pages 39-54 in: Detection and Diagnostics of Plant Pathogens. L. M. Gullino and M. P. J. Bonants, eds. Springer Netherlands, Dordrecht, The Netherlands.

Mahaffee, W. F., and Stoll, R. 2016. The ebb and flow of airborne pathogens: Monitoring and use in disease management decisions. Phytopathology 106: 420-431.

Mori, Y., Nagamine, K., Tomita, N., and Notomi, T. 2001. Detection of loopmediated isothermal amplification reaction by turbidity derived from magnesium pyrophosphate formation. Biochem. Biophys. Res. Commun. 289:150-154.

Notomi, T., Okayama, H., Masubuchi, H., Yonekawa, T., Watanabe, K., Amino, N., and Hase, T. 2000. Loop-mediated isothermal amplification of DNA. Nucleic Acids Res. 28:e63.

Pinnschmidt, H., Klein-Gehbinck, H., Bonman, J., and Kranz, J. 1993. Comparison of aerial concentration, deposition, and infectiousness of conidia of Pyricularia grisea by spore-sampling techniques. Phytopathology 83:1182-1189.

Poon, L. L. M., Wong, B. W. Y., Ma, E. H. T., Chan, K. H., Chow, L. M. C., Abeyewickreme, W., Tangpukdee, N., Yuen, K. Y., Guan, Y., Looareesuwan, S., and Peiris, J. S. M. 2006. Sensitive and inexpensive molecular test for Falciparum malaria: Detecting Plasmodium falciparum DNA directly from heattreated blood by loop-mediated isothermal amplification. Clin. Chem. 52:303-306.

Rossman, A. Y., Howard, R. J., and Valent, B. 1990. Pyricularia grisea, the correct name for the rice blast disease fungus. Mycologia 82:509-512.

Thiessen, L. D., Keune, J. A., Neill, T. M., Turechek, W. W., Grove, G. G., and Mahaffee, W. F. 2016. Development of a grower-conducted inoculum detection assay for management of grape powdery mildew. Plant Pathol. 65:238-249.

Tomlinson, J. A., Barker, I., and Boonham, N. 2007. Faster, simpler, more-specific methods for improved molecular detection of Phytophthora ramorum in the field. Appl. Environ. Microbiol. 73:4040-4047.

Tomlinson, J. A., Ostoja-Starzewska, S., Adams, I. P., Miano, D. W., Abidrabo, P., Kinyua, Z., Alicai, T., Dickinson, M. J., Peters, D., Boonham, N., and Smith, J.
2013. Loop-mediated isothermal amplification for rapid detection of the causal agents of cassava brown streak disease. J. Virol. Methods 191:148-154.

Tosa, Y., Uddin, W., Viji, G., Kang, S., and Mayama, S. 2007. Comparative genetic analysis of Magnaporthe oryzae isolates causing gray leaf spot of perennial ryegrass turf in the United States and Japan. Plant Dis. 91:517-524.

Uddin, W., Serlemitsos, K., and Viji, G. 2003a. A temperature and leaf wetness duration-based model for prediction of gray leaf spot of perennial ryegrass turf. Phytopathology 93:336-343.

Uddin, W., Viji, G., Schumann, G. L., and Boyd, S. H. 2003b. Detection of Pyricularia grisea causing gray leaf spot of perennial ryegrass turf by a rapid immuno-recognition assay. Plant Dis. 87:772-778.

Uddin, W., Viji, G., and Vincelli, P. 2003c. Gray leaf spot (blast) of perennial ryegrass turf: An emerging problem for the turfgrass industry. Plant Dis. 87 880-889.

Viji, G., Wu, B., Kang, S., Uddin, W., and Huff, D. R. 2001. Pyricularia grisea causing gray leaf spot of perennial ryegrass turf: Population structure and host specificity. Plant Dis. 85:817-826.

Villari, C., Tomlinson, J. A., Battisti, A., Boonham, N., Capretti, P., and Faccoli, M. 2013. Use of loop-mediated isothermal amplification for detection of Ophiostoma clavatum, the primary blue stain fungus associated with Ips acuminatus. Appl. Environ. Microbiol. 79:2527-2533.

Vincelli, P., and Dixon, E. 2002. Resistance to QoI (strobilurin-like) fungicides in isolates of Pyricularia grisea from perennial ryegrass. Plant Dis. 86: 235-240.

Vincelli, P., Dixon, E., and Farman, M. 2008. Susceptibility of selected cultivars of forage grasses to Magnaporthe oryzae isolates from annual ryegrass and relatedness of the pathogen to strains from other grasses. Online publication. Forage Grazinglands 6.

West, J. S., Atkins, S. D., Emberlin, J., and Fitt, B. D. L. 2008. PCR to predict risk of airborne disease. Trends Microbiol. 16:380-387. 